\title{
THE IMPACT OF INFORMATION TECHNOLOGIES ON THE SCOUTING PROCESS IN SPORTS GAMES
}

\author{
Srđan Marković, \\ Ivan Ćuk, \\ Aleksandar Živković \\ Faculty of Physical Education and Sports \\ Management, \\ Singidunum University, \\ Belgrade, Serbia
}

\begin{abstract}
:
Although it has attracted the attention of experts only in the last decade, sports analytics has its roots since the existence of organized sports. Any records of players or situations in the game that contribute to later decisionmaking during the competition can be considered as some kind of analytics. As an important part of these processes, scouting is at the core of coaching in sport games at all levels of competition. Coaches and managers try to enable improvements in competitive activity by exploring the key factors and mechanisms on which success in competition depends, both at the individual level as well as at the level of the entire team. The aim of this paper is to describe how technological development and ultimately information technology have influenced these processes, and specifically how modern tendencies affect performance management in sports games. Primarily, the development of data collection methods, from manual, through video, to the use of modern digital technology has led to an "explosion" of the amount of data collected. The huge amount of available data has resulted in the emergence of expert data systems specific to each sport game, which provides analytical support to coaches and managers in decision-making.
\end{abstract}

Keywords:

decision, analytics, feedback, system, data.

\section{INTRODUCTION}

Participation in sports is usually undertaken with the intention of improving the level of activity. One of the most important variables that influence learning and later performance of a skill is feedback. In order to provide meaningful feedback, the coach must somehow observe and evaluate performance $[1,2]$. Practice in coaching includes conclusions and subjective observations on coaching perception, bias, and previous experiences. However, numerous studies have found that this kind of observations could be incorrect and unpredictable. Memory capacities in humans are somehow limited and it is nearly impossible for us to remember in details all the important events that happened during the entire match.
Correspondence:

Srđan Marković

e-mail:

smarkovic@singidunum.ac.rs 
So far, it was shown that top football coaches remembered just $30 \%$ of the key factors of successful football performances and were less than $45 \%$ accurate in their post-match assessment $[3,4]$. In fact, more experienced coaches even reported a non-existent difference in performance. In addition, they were very confident in their decisions, even when they were making mistakes [5].

Considering how people process information, the aforementioned results are not surprising. Memorizing certain information and retrieving it later is a complex process with great potential for interference. Impressive parts of the competition (e.g. controversial decisions, exceptional technical performances, free kicks, etc.) are easy to remember, while less impressive moments are mostly forgotten. Moreover, all actions that take place in the field are almost impossible to view, assimilate and store, due to the limitation in our system. As a result, coaches are generally focused on just a few critical parts, while other actions generally remain neglected $[1,5,6]$.

Surprisingly, it appears that standard or predefined system for monitoring and evaluating performance does not exist. If reliance on a human information processing system is problematic, another way of gathering information during sports competitions must be determined. It is obvious from the previous text that this information must be objective, unambiguous and as comprehensive as possible. Therefore, the aim of this paper is to describe how technological development and ultimately information technology have influenced these processes, i.e. how modern tendencies affect performance management in sports games.

\section{METHODS}

For the purposes of this study, a bibliographicdescriptive method was used. Literature search was conducted with following services: PubMed, Google, Google Scholar and Kobson. The keywords for searching the appropriate literature were: sport games, analytics, scouting, technology, and data.

\section{RESULTS AND DISCUSSION}

Technological development has improved the data collection systems during the competition and made it easier for statistical analysis. The emergence of video, and then digital technology, has been shown to have two effects on the type of activity data collected. First, there is much more actions on which data can be collected. Second, $g$ of the nature of data collected by technological advances change the possibilities on data analysis, providing the means to collect data on deeper aspects of activities. The analysis of competitive activity includes not only the frequency of different types of actions performed, but also data on the more fundamental dimensions of performance on which actions are observed. These more fundamental aspects of performance can be summarized as technique, tactics, fitness and psychology. The impact of technology on the collection of data on athlete activities is discussed using a three-step scheme: (a) manual method, (b) video analysis and (c) digital technology $[7,8,9]$.

Regardless of the level of technology, player tracking systems had to provide data on four key elements of the analysis. The basic key elements in system analysis are PLAYER, POSITION, ACTION AND TIME [1]. Each advanced system improved the data collection of the previous system.

\section{Phase 1 - Manuel method}

The manual method, as the oldest and unavoidable method of data collection, is a suitable system for structurally simple sports games such as baseball, tennis, and volleyball to some extent.

The structure of the game, divided into a series of individual parts, facilitates the collection of key data on activities during the game. The game is played according to the rules of the break between points, the ball is returned to the server, which starts a new segment. That way, there is enough time to record the basic details of the past activities. In addition, this game is viewed from two angles: the angle of defense and the angle of attack. Although this method is effective in sports games such as baseball, its use is limited in sports games with the conquest of space, such as football, basketball, handball, ice hockey or rugby.

Scatter diagrams are frequently used to collect data during a match and provide instantaneous feedback athletes and coaches. They are usually used in the form of drawings on the scheme of the field of the sports game that is observed, and then actions of interest are recorded on the positions that have taken place [1]. For example, consider a football coach who wants to know where his team is losing possession (Fig. 1a). Second, coach wants to know which player loses the most balls (Fig. 1b).Third, maybe the most important are the actions that players perform when losing possession. In order to record actions, it is necessary to define the most common actions and assign them a symbol: $\mathrm{P}$ - pass, $\mathrm{S}$ - shot, D - dribble, C - cross, $\mathrm{K}$ - kick-off. This level is the most complicated and requires the most practice (Fig. 1c). 
a)

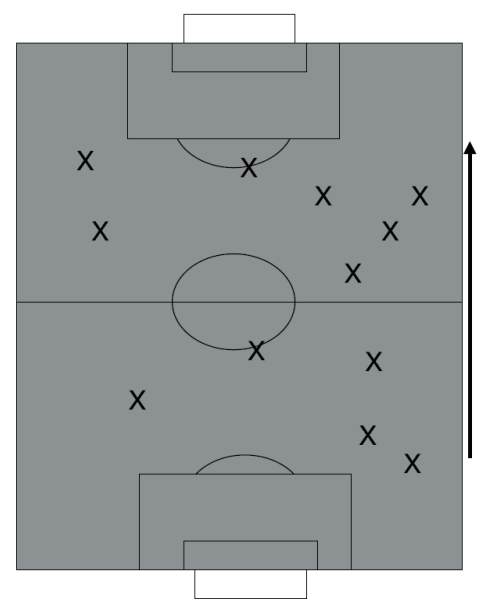

b)

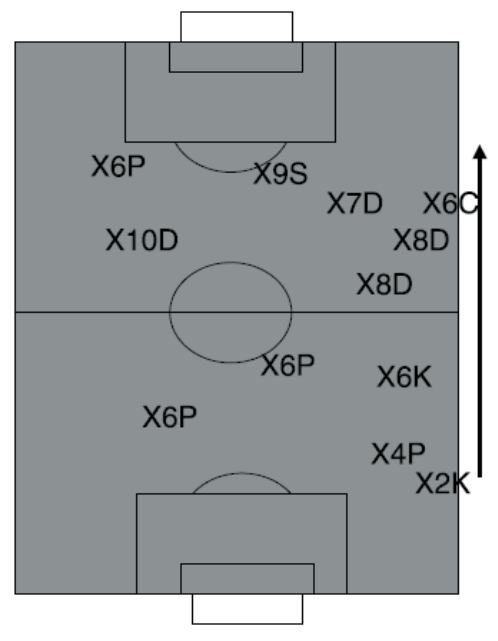

c)

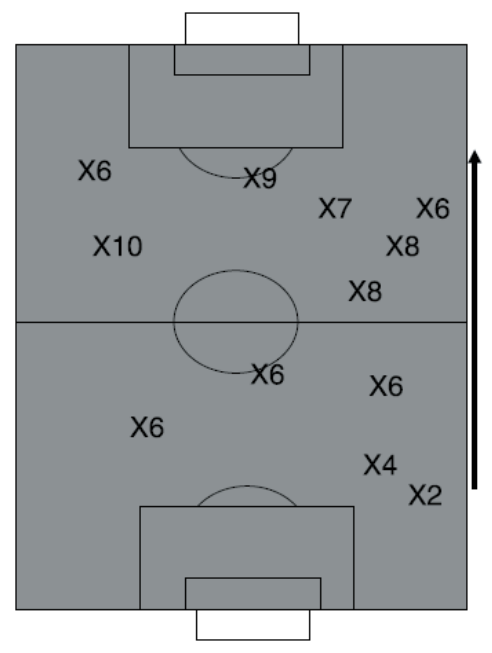

Fig. 1. Scatter diagrams in football

Frequency tables are another common form of data collection that allows quick, easy analysis of the activities of athletes and teams. Using a frequency table (Fig 2) below, the analyst can easily record the frequency of each player's action in the line-up durng basketball game [1].

\begin{tabular}{|c|c|c|c|c|c|}
\hline Actions & $1 \quad 2$ & 3 & 4 & 5 & 6 \\
\hline Pass & 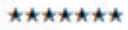 & $\star \star \star$ & $\star \star \star \star \star$ & & \\
\hline Dribble & $\star \star \star$ & $\star$ & $\star \star$ & & \\
\hline 2-pt Shot & $\star \star \star \star \star$ & $\star \star \star \star$ & $\star \star$ & & \\
\hline 3-pt Shot & $\star \star$ & & $\star$ & & \\
\hline Assist & $\star \star \star \star \star$ & $\star \star \star \star \star$ & $\star$ & & \\
\hline Lost possession & $\star$ & $\star \star$ & & & \\
\hline
\end{tabular}

Fig. 2. Frequency table for basketball

The main characteristics of these systems are:

- Easy and fast

- Precise (with proper practice)

- Used during competition

- Gives instant feedback

- Efficient, no need for analysis

- No sequence of events.

It must be noted that there are dangers in the interpretation these data. Namely, not only that they lack in accuracy, they can be only analyzed by the simplest procedures. Attempts at in-depth analysis with this kind of data only lead towards problems $[1,2,10]$.

\section{Phase 2 - Video recording}

The main characteristic of sports games with the conquest of space is the attempt of the team to move through the space to get into a situation to place a certain object in a certain place (basket, goal) that is defended by the opposing team. Additionally, these games are time-dependent, i.e. the result of the match is determined by the cumulative result for a certain time. The main feature of these games is tactics and all players on the field are directly or indirectly involved in every action. Consequently, players in decision-making during the match must be both spatially and tactically aware [7].

In comparison to the baseball or tennis, in more complex sport games, a much more compound structure is noticed. Specifically, there is a much more indirect connection between the individual contribution of the players, the achievement of goals and the results of the match. Attack and defense are much more interdependent, because the possession of the ball by the attacking team also has a defensive function (e.g. depriving the opposing team of opportunities). Therefore, ball possession and time are key resources in these sport games. For example, in order to score a goal, the ball must move forward into areas of the field where opportunities to score can be created, thus proving a high interdependence of different attack actions. 
This structural hierarchy of interdependent player actions creates serious problems for analysts, esspecially when there are joint actions $[7,10,11]$.

Not surprisingly, the manual method can only collect limited amounts of data during sports games. Appearances, results, disciplinary warnings and player exclusions are the only data consistently recorded through the history. Game recordings greatly facilitated the collection of data on activities in sports games with the conquest of space and enabled more detailed analysis. Instead of limiting themselves to collecting data during the match collect data by applying the manual method to video reproductions of matches (Fig. 3). This process was very slow and time consuming. As a result, the teams were focused only on the critical moments. Therefore, the video analysis was mostly qualitative with reconstructions of certain parts, often only those in which the result changed. Quantitative data for these games proves to be quite restricted in range [7].

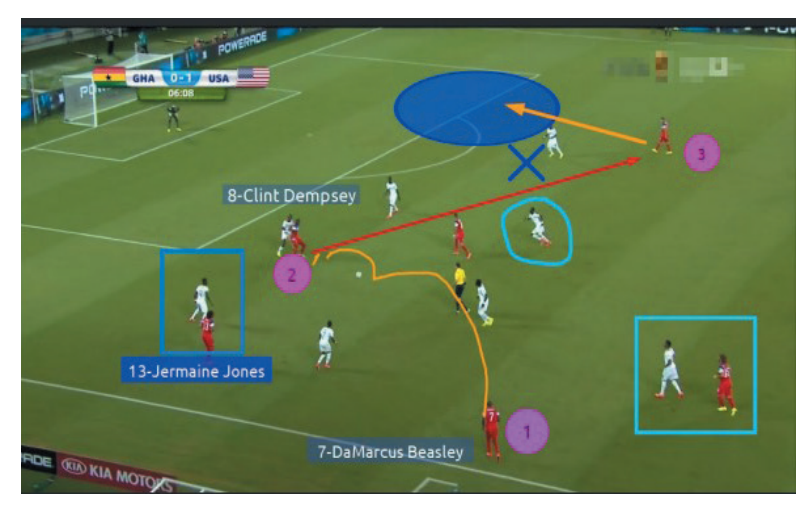

Fig. 3. Video analysis in football

\section{Phase 3 - Digital technology}

The arrival of digital technology and cheap computers increased the speed of data collection in various sport games. Digitization of video images enabled the development of computer software for encoding and editing videos, so that the process of data collection became much faster. Specifically, image recognition software became somehow automated, with limited human intervention only. Due to digital technology, data is available for all actions of different players in spaceconquering sports games $[1,7,12-14]$

The digital revolution in activity analysis has led a number of commercial companies to offer data collection and analysis services to a certain number of sports teams. All of these systems provide detailed numerical data on all aspects of the game.
They combine numeric data with graphics and video from the game. These systems also allow a certain degree of interactivity so that the analyst can control some parameters when reporting [7]. Usually teams rely on these systems to get detailed statistical reports after the game, as a source of data on future opponents and scouting reports to recruit players (Fig. 4). Additionally, teams often try to create databases on their own (e.g. SportsCode, InStat etc.) to encode and edit footage of their matches. Because of the time pressures that are quite evident, analysts can encode only several real time features. As a result, the focus is merely on the important data that can put some pressure into the tactical changes $[2,10,14]$.
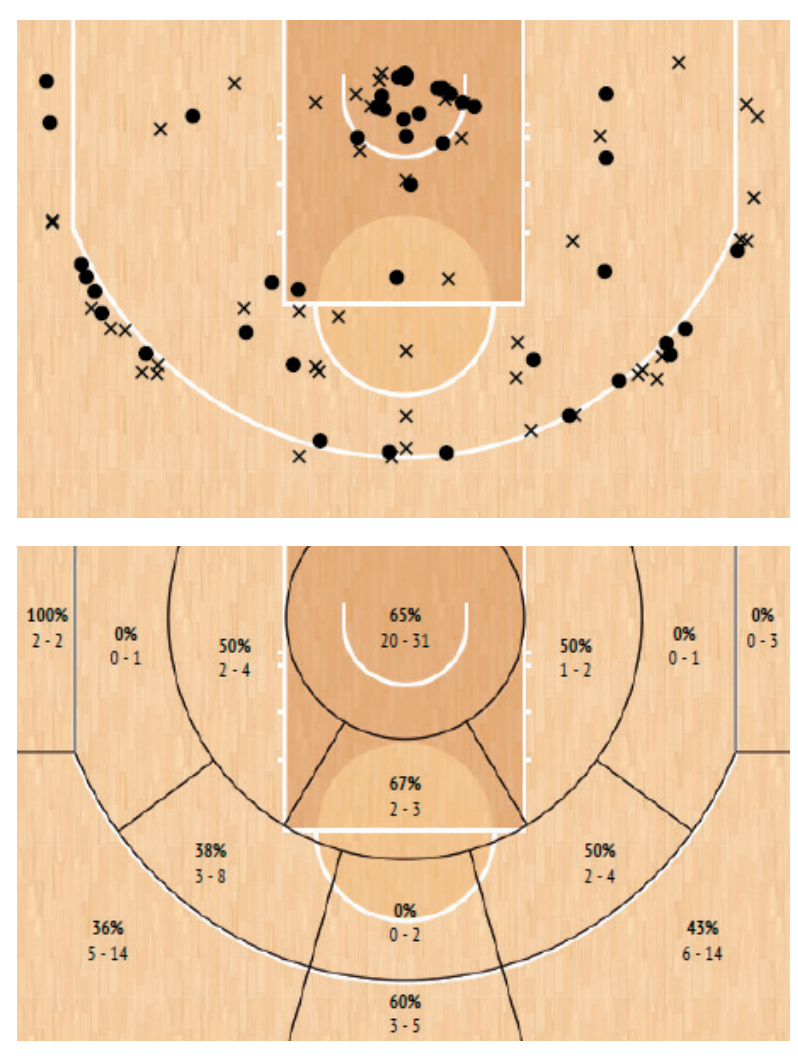

Fig. 4. Part of InStat basketball game report

The digital systems development has led to the immense expansion in numerical data provision, as well as to the availability of activity tracking data (including the continuous data on the player's position). Primarily, these types of data were obtained by a multiple cameras triangulation in order to place the precise player's positions. This provided data on the sections covered and, in combination with the weather data, the speed at which the players move. Finally, the teams had obtained consistent data on at least one feature of the players' physical activities throughout the matches $[7,11,14]$. 
The use of global positioning systems (GPS) proves to be an alternative approach to the video activity tracking systems.. Recently, the most of sports games have included GPS devices to obtain activity tracking data. Nevertheless, probably the most ground-breaking utilization of GPS devices (Fig. 5) is tracking sections covered and speeds in training [7, 11-13].

\section{Future (or present) - Expert data systems}

Methods for collecting sports data has continuously advanced, thus allowing the teams to quickly encode and edit match videos by themselves. That led to creating the possibility data systems in which data are defined and interpreted by trainers, and do not rely on commercial providers (i.e. expert data systems). That way, trainers can evaluate choice of actions (decisions) as well as their execution (itechnique). In general, that refers to "doing the right things" (choosing) or "doing things right" (doing).

An important component of tactical decision-making in sports games is the spatial positioning of players. Players constantly have to make decisions about where to position themselves when their team attacks and defends. For instance, attackers must supply with opportunities for the player in possession. That can be obtained by moving to the receiving positions of the ball or by making movements to create space. Defensive players, on the other hand, must make position decisions to face a particular attacking player or some exact field part. In addition, tracking technology has greatly helped coaches assess players 'decisions about a position $[10,11,15]$.

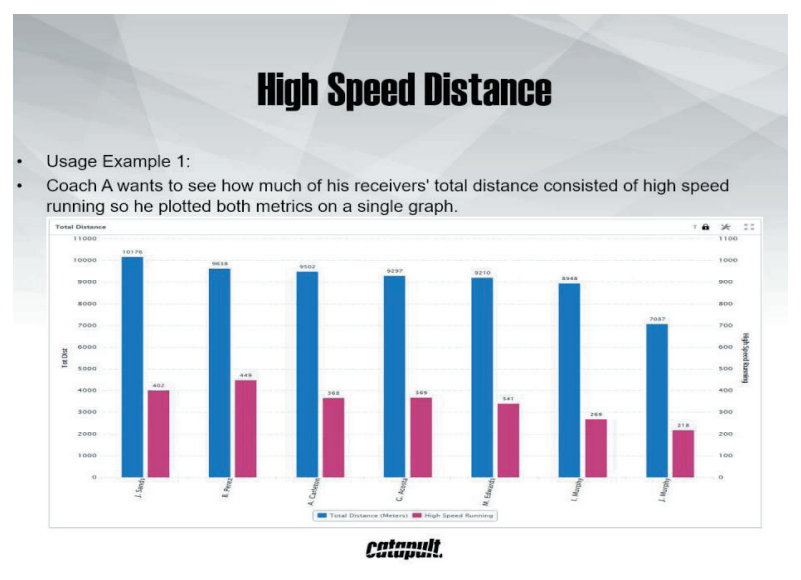

Fig. 5. Part of Catapult system report

\section{CONCLUSIONS}

The technology impact on data collection in professional sport has provided an exponential increase in the amount of collected data, which inevitably amplified the requirement for qualitative to quantitative data analysis. Qualitative data analysis is complemented by a quantitative analysis - initially by summarizing statistics and, by using other quantitative techniques, such as statistical analysis to analyze models in different activities. Technological development sport related data acquisition, has quite considerably influenced sports games through a dominant conquest of space, which has really become possible with the advent of digital and information technology.

\section{REFERENCES}

[1] M. Hughes and I. Franks (Eds.), "Notational Analysis of Sport", London and New York: Routledge, 2004.

[2] MD. Hughes and RM. Bartlett, „The use of performance indicators in performance analysis", Journal of Sports Sciences, vol. 20, pp. 739-754, 2002.

[3] IM. Franks and G. Miller, „Eyewitness testimony in sport”, Journal of Sport Behavior, vol. 9, pp. 39-45, 1986.

[4] IM. Franks and G. Miller, „Training coaches to observe and remember", Journal of Sports Sciences, vol. 9, pp. 285-297, 1991.

[5] IM. Franks, „The effects of experience on the detection and location of performance differences in a gymnastic technique", Research Quarterly for Exercise and Sport, vol.64, no. 2, pp. 227-231, 1993.

[6] IM. Franks, D. Goodman, and G. Miller, "Analysis of performance: Qualitative or Quantitative?". SPORTS, March, 1983.

[7] B. Gerrard, "Analytics, Technology and High Performance Sport”. In: Critical Issues in Global Sport Management, N. Schulenkorf and S. Frawley, Eds. London and New York: Routledge, pp. 205-218, 2017.

[8] P. O'Donoghue, (Ed.), "Research methods for sports performance analysis". London: Routledge, 2010.

[9] C. Carling, MA. Williams and T. Reilly, "Handbook of soccer match analysis", London and New York: Routledge, 2005.

[10] L. Nelson and R. Groom, "The analysis of athletic performance: some practical and philosophical considerations", Sport, Education and Society, vol. 17, no. 5, pp. 687-701, 2012. 
[11] "5 steps to success in video analysis", Cataput E-books, Downloaded 15.02.2020. from https:// go.catapultsports.com/five-steps-to-success-videoanalysis

[12] RA. Den Hartigh, MS. Niessen, WGP. Frencken and RR. Meijer, "Selection procedures in sports: Improving predictions of athletes' future performance", European Journal of Sport Science, vol. 18, no. 9, pp. 1191-1198, 2018.

[13] H. Sarmento, R. Marcelino, MT. Anguera, J. Campanico, N. Matos and JC. Leitao, "Match analysis in football: a systematic review", Journal of Sports Sciences, vol. 32, pp. 1831-1843, 2014.
[14] InStat reports. Downloaded 15.09.2020. from https://instatsport.com/basketball/instat_reports

[15] "5 steps to success in athlete monitoring", Cataput E-books, Downloaded 15.02.2020. from https:// go.catapultsports.com/five-steps-to-success-athletemonitoring 